\title{
Improving RWA-OBS Formulation and Solution
}

\author{
Thomas Coutelen \\ ECE, Concordia University \\ Montreal, Qc, H3G 1M8, Canada \\ Email: t_coutel@ece.concordia.ca
}

\author{
Brigitte Jaumard \\ CIISE, Concordia University \\ Montreal, Qc, H3G 1M8, Canada \\ Email: bjaumard@ciise.concordia.ca
}

\author{
Gérard Hébuterne \\ Telecom-SudParis \\ Evry, France \\ Email:gerard.hebuterne@it-sudparis.eu
}

\begin{abstract}
Loss-less transfers are commonly associated with circuit switching: Circuit switching in all-optical networks (OCS) prevent from collisions by avoiding multiplexing below the wavelength granularity. Spectral multiplexing can be combined with time multiplexing in opaque networks at the expense of additional equipment (MSPP) and complex synchronization issues. Optical Burst Switching has been proposed a decade ago as a promising alternative to OCS: Its asynchronous nature and its arbitrary granularity open the possibility of statistical multiplexing, but it is not well suited to handle loss-less requirements. Wavelength routing and TDM can still be performed at the expense of a slight modification of the control plane, but those solutions override the OBS flexibility and avoid the statistical multiplexing.

The RWA-OBS problem tends to solve the drawbacks of both WR-OBS and SOBS. Based on several properties related to the in advance reservation and the data plane transparency of $O B S$, it integrates particular cases where two flows can be multiplexed on a given wavelength in a genuine OBS fashion without loss. We first enhance the original RWA-OBS formulation and then describe an iterative greedy heuristic and a column generation approach to improve the computing time and the scalability of the model. Experiments are conducted on two topologies to illustrate the potential of RWA-OBS that can compete with SOBS without requiring synchronization. The new proposed methods are then compared in terms of computing times and qualities. The efficiency of the column generation approach allows the integration of an additional constraint to control the compromise between the burst insertion delay and the throughput.
\end{abstract}

\section{INTRODUCTION}

The OBS popularity fluctuated since its emergence [1] a decade ago. The infatuation of this promising technology declined, mainly because of the difficulty to achieve loss-less transfers. In OBS, contentions can occur even under a light load and still today, no mechanism, either in space, time or spectral domain can ensure that all contentions can be solved [2]. Proactive mechanisms such as load balancing can reduce the contention rate (e.g., [3], [4]), but pure OBS loss-less configuration has not yet been achieved.

Polymorphous OBS (POBS, [5]) describes a slightly modified control plane that enables circuit switching at a wavelength (WR-OBS) and sub-wavelength (SOBS) granularity together with opportunistic transfers in a genuine OBS fashion. Circuit transfers can handle loss-less requirements but WROBS avoids statistical multiplexing and suffers from its coarse granularity (similarly to OCS, see [6]). Optical grooming can be achieved to reduce the granularity of WR-OBS (see, e.g., [7]). This technique is however not limited to WR-OBS and can be adapted to our proposal. SOBS solves the granularity issue by multiplexing several sub-wavelength connections in a TDM manner, but it introduces several complex synchronization issues (see, e.g., [8]-[10]). SOBS has been successfully applied in [11] where the authors restructure the network topology around a set of interconnected clusters.

Both WR-OBS and SOBS avoid loss by preventing contentions. In OBS networks however, the streamline effect [3] and the offset time priority [12] disclose particular cases where a burst is ensured to survive potential contentions. As a result, a kind of statistical multiplexing can be done asynchronously without loss.

The streamline effect emanates from the data plane transparency: The bursts are signaled in-advance by a header so that the switches can be configured before the burst arrival. It entails that the delay between two successive bursts remains unchanged as long as they use the same path. Consequently, as two bursts entering a given input port cannot overlap, they cannot contend for any output port (see [13]).

Contentions thus only occur between bursts arriving from different input ports and requesting the same output port for overlapping time periods. If available contention resolution mechanisms fail, only the first signaled burst will survive (regardless of its effective arrival date). What matters is the header arrival time, largely decided by the offset time $(O T)$ assigned to the burst. The $O T$ introduced by the JET reservation protocol [1] solves the lack of optical memory in the data plane: The header is responsible for resource reservation and is sent ahead of the payload so that it can be converted, processed and re-emitted in the optical domain before the burst arrival. The $O T$ budget is spent while going through each intermediate node. As a result, the burst to be dropped (the latest signaled) is more likely to be the closest one to its destination (the one with the smallest $O T$ ). In [12] and [14], an extra offset time is used in order to achieve service differentiation. The mechanism performs isolation of bursts of a given priority over bursts of lower priority. Nevertheless, the mechanism only provides control on the flows that are affected by the loss but does not avoid loss.

Mutual isolation between two flows can be achieved by a careful use of the electrical buffering at the network entrance. The ALAP MAC protocol described in [15] ensures that an isolated transit burst survives a contention with an ingress burst. As the ingress burst is still in electrical domain, it also survives by accessing electrical buffering. The idea is to delay the ingress burst reservation as late as possible so 
that transit bursts header precedes the contention discovery. In some configurations disclosed in [15], the access delay is significantly increased, but the end-to-end delay is still reduced as then we rule out retransmission mechanisms.

The RWA-OBS problem was first introduced in [15] and performs routing and wavelength assignment. Contrary to RWA for OCS (see, e.g., [16]) or WR-OBS, ingress flows and transit flows can be merged if the transit flows are $O T$-isolated and the ingress nodes run ALAP. The streamline effect then avoids further contention between the bursts of those flows. As a result, the granted flows have loss-less guarantee.

In [15], it is shown that extending the $O T$ improves the loss-less multiplexing potential and, consequently, the grade of service. The formulation described in [15], however, assumes a constant $O T$ extension factor $(E O T)$ for all flows. In this paper, we introduce the flexibility to use different $E O T$ for each connection. To handle the model growth and improve its scalability we propose two solution mechanisms: A greedy heuristic and a column generation formulation combined with a Tabu search algorithm. Note that the ALAP burst insertion protocol can affect the delay. Our model integrates an additional set of constraints that controls the compromise between the delay and the throughput.

The paper is organized as follows. Section II presents the streamline effect, the offset based priority and the ALAP burst insertion protocol required for mutual isolation. Section III presents the isolation and mutual isolation patterns. Section IV describes our improved RWA-OBS model and a greedy heuristic. The column generation formulation is presented in Section V. Experiments are reported in Section VI. Conclusions are drawn in Section VII.

\section{OBS PROPERTIES}

\section{A. Streamline Effect}

The streamline effect relies on the observation that connections arriving on the same input port cannot contend. In the example illustrated in Figure 1, $C_{1}$ and $C_{3}$ can contend for the link $2 \rightarrow 3$, but the bursts that successfully access this link cannot contend for link $3 \rightarrow 4$, whatever the connection they belong to. Note the particular case of $C_{2}$ that merges with $C_{1}$ and $C_{3}$ on its first link. In that situation, electrical buffers can store bursts of $C_{2}$ to delay their emission and solve the contention.

The streamline effect is deeply investigated in [3], where the authors propose a reasonable approximation of the loss for multi-rate flows, as long as the number of sources remains small. Note that the Engset loss formula (see, e.g., [17]) also successfully reflects the streamline effect, whereas the ErlangB loss formula should be rejected. These points are further discussed in [13].

\section{B. Offset Time Priority}

Contention occurs if a header requests an output port for a time slot that is not completely free. In the absence of contention resolution mechanism, the latest signaled burst is dropped. The offset time $(O T)$ plays an important role in the

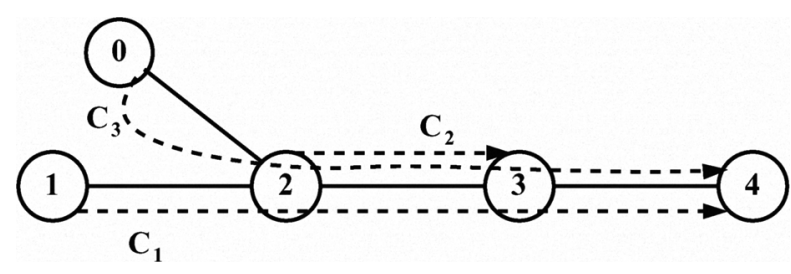

Fig. 1. contention

dropping process. In [15], a loss evaluation adjustment that takes into account the respective $O T$ of the contending bursts is proposed. For the scope of this paper, we will only focus on the case where the $O T$ protects a connection, i.e., the case where all dropped bursts belong to the same connection. It is illustrated by Figure 2 . Burst $B_{1}$ is signaled by header $H_{1}$ at time $t_{1}$. Let us consider burst $B_{2}$ of duration $b_{2}$ signaled by header $H_{2}$ at time $t_{2}$ such that $O T_{B_{1}}-O T_{B_{2}}=b_{2}$. In that case, no contention can occur if $H_{2}$ arrives before $H_{1}$ (Figure 2-C). Indeed, in case of contention, $\mathrm{H}_{2}$ necessarily arrives after $H_{1}$ and $B_{1}$ cannot be dropped. More formally, $B_{1}$ is dropped if the bursts contend and $H_{2}$ arrives before $H_{1}$, i.e., if $t_{1}>t_{2}$ and $t_{2}+O T_{B_{2}}+b_{2}>t_{1}+O T_{B_{1}}$. The two conditions can be rewritten as follows: $0<t_{1}-t_{2}<$ $b_{2}-\left(O T_{B_{1}}-O T_{B_{2}}\right)$ to highlight the fact that $B_{1}$ cannot be dropped if $\left.O T_{B_{1}}-O T_{B_{2}}\right)>b_{2}$. Although the $O T$ should depend on both the route and the class of service of the bursts, in this paper, we assume that all bursts traveling on a given route have the same $O T$, computed as follows:

$$
O T_{r}=\delta \times \ell_{r} \times \alpha_{r}
$$

where $\delta$ is the header processing time, $\ell_{r}$ is the number of hops on $r$ and $\alpha_{r} \geq 1$ is an $O T$ extension factor, used to increase the priority of the bursts on $r$ (similarly to the extra offset time used in, e.g., [14], but in a multiplicative way). As a sequel, at a given node, $B_{1}$ is isolated from $B_{2}$ if $\ell_{r_{1}} \alpha_{r_{1}}-\ell_{r_{2}} \alpha_{r_{2}}>$ $b_{2} / \delta$.

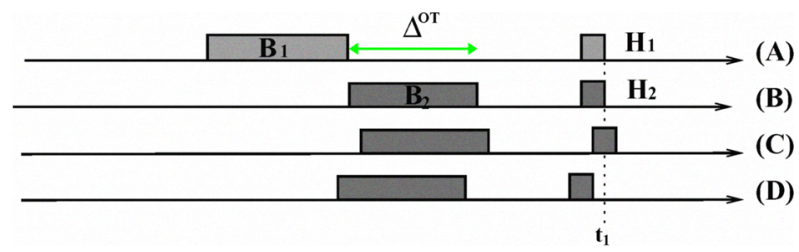

Fig. 2. OT priority

\section{ALAP protocol}

If a burst contends for its first link, it is still in electrical domain and can be stored in electrical buffers in order to survive from the contention. The electrical buffering, however, can increase the offset time of the ingress burst if not managed properly. A side-effect is that isolation state of transit flow may be broken. The ALAP protocol consists in delaying both the burst and the reservation attempt so that the $O T$ of the 
ingress burst is not perturbed by electrical storage (see [13] for more details). Figure 3 illustrates the ALAP protocol, formally described by Algorithm 1.

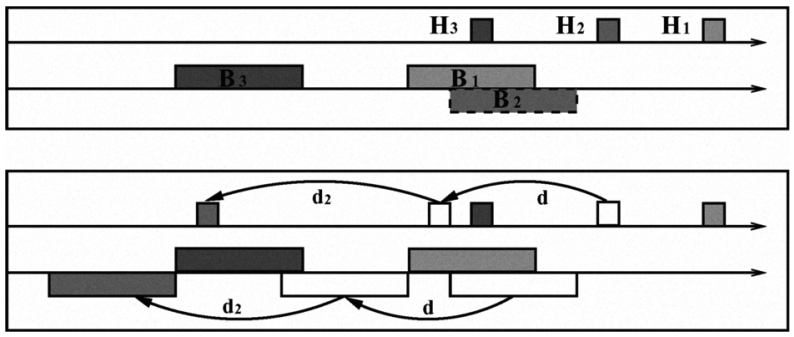

Fig. 3. The ALAP Burst Insertion

Burst $B_{2}$ is aggregated and ready to be launched at time $t_{2}$. The output port is requested from $t_{2}^{\prime}=t_{2}+O T_{r_{2}}$ to $t_{2}^{\prime}+b_{2}$. As the output port has already been reserved by $H_{1}$ for an overlapping period of time, $B_{2}$ emission must be delayed in electrical buffers to solve the contention. The ALAP protocol consists in delaying the reservation attempts as well so that, in case of contention with further transit bursts, the ingress burst is the latest signaled and electrical buffering can be reused. In our example, the reservation for $B_{2}$ is postponed by $d$ time units. This way, the ingress node keeps the control on $O T_{r_{2}}$ and the isolation of $B_{3}$ is respected: $H_{3}$ arrives before the next reservation attempt of $B_{2}$ and $B_{3}$ can be served. This contention can be handled in the same way: $B_{2}$ reservation is postponed again by $d_{2}$ units of time and no burst is lost.

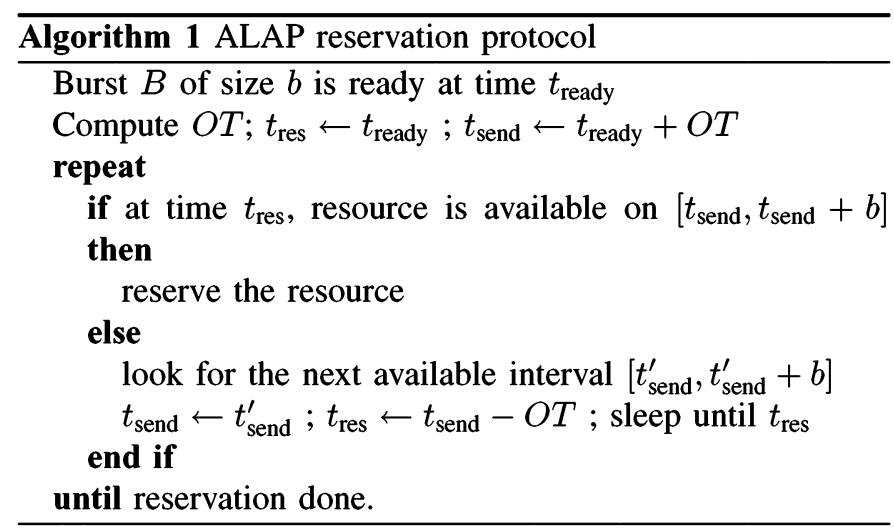

The impact of the ALAP burst insertion protocol has been evaluated in [15]: The simulation involves a source of traffic whom bursts contend with transit bursts. The ingress node runs ALAP and the measured average insertion delay of ingress bursts is reported on Figure 4 . The $x$-axis represents the proportion of ingress traffic in the overall load. The increase of insertion delay due to ALAP protocol is negligible if the ingress traffic dominates the transit traffic. Otherwise, the increase of the insertion delay remains reasonable for an overall load above 0.6 Erlang. By the way, the impact of the insertion delay must be compared with the end-to-end delay achieved with retransmission of the dropped bursts.

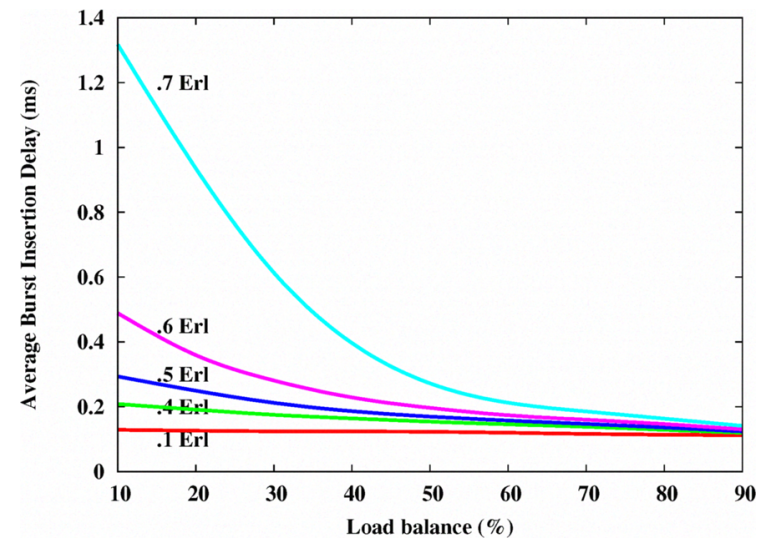

Fig. 4. Impact of ALAP on burst insertion delay

\section{FLOW ISOLATION}

\section{A. General Case}

Route $r$ is isolated from route $r^{\prime}$, denoted by $r \triangleright r^{\prime}$, if no burst of $r$ can be lost due to bursts of $r^{\prime}$. Thanks to the streamline effect, the isolation has only to be checked on the merging nodes (i.e., the nodes on which the routes converge). We have identified three isolation configurations. Firstly, link disjoint routes are isolated since they never converge. Secondly, a route is isolated on its first link as bursts can be stored in electrical buffers at their source nodes. Finally, the offset time priority discloses an additional isolation configuration as described in section II-B. We denote by $O T_{r_{i}}^{v}$ the remaining $O T$ value of a burst on route $r_{i}$ at node $v$. For given routes $r_{i}$ and $r_{j}$, we define $\Delta_{r_{i}, r_{j}}^{O T}=\min _{v \in V}\left(O T_{r_{i}}^{v}-O T_{r_{j}}^{v}\right)$, where $V_{i j}$ is the set of nodes where routes $r_{i}$ (carrying $B_{i}$ ) and $r_{j}$ (carrying $B_{j}$ ) merge. By convention, $\Delta_{r_{i}, r_{j}}^{O T}=\infty$ if $r_{i}$ and $r_{j}$ are linkdisjoint or if $V_{i j}$ is reduced to the first node of $r_{i} . r \triangleright r^{\prime}$ if $\Delta_{r, r^{\prime}}^{O T}>b_{r^{\prime}}$, where $b_{r^{\prime}}$ is the size of the bursts on route $r^{\prime}$. Figure III-A illustrates this case. $C_{1}$ competes with $C_{2}$ at node 2. Destination of $C_{2}$ is more distant than the destination of $C_{1}$ so, without extra offset time, $\Delta_{r_{2}, r_{1}}^{O T}>0$. If $\Delta_{r_{2}, r_{1}}^{O T} \geq b_{1}$, then $C_{2} \triangleright C_{1}$.

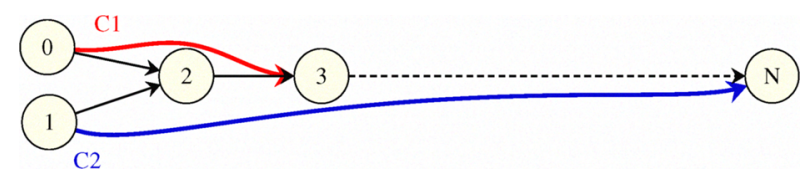

Fig. 5. General Isolation Case: Simple Bus Topology

Note that if $\delta>b_{1}$, then $C_{2} \triangleright C_{1}$ if $r_{2}$ is at least one hop longer than $r_{1}$. This is the most favorable case for maximizing the multiplexing potential of RWA-OBS. Although this assumption has been suggested in [12], it is worth to discuss its relevance at the light of recent contributions.

Several arguments suggest the use of small bursts for the purpose of the aggregation delay, the contention resolution (the efficiency of the FDLs is improved with smaller bursts, 
see [18]) or the goodput of TCP connections. In [19], authors shows that spreading the incoming load among several burstifiers (i.e., burst aggregation queues) reduces the impact of the synchronized loss on the goodput of TCP sources, but it mandates to reduce the size of the bursts to avoid long burstification time.

Opposite arguments can be set forth. Indeed, longer bursts reduce the signaling overhead and intensify the traffic shaping. This contributes, in turn, to reduce the contention rate in the core network (see [15], [20]).

All those arguments apply in some specific contexts, but the following one is valid independently of the equipment or protocols: The guard time between successive bursts, required to reconfigure the switch, links the size of the bursts with the maximal wavelength utilization and thus imposes a lower bound on the size of the bursts. In [21], the header processing time $\delta$ is around $50 \mu \mathrm{s}$, whereas the reconfiguration time is evaluated around $50 \mathrm{~ns}$ in [22]. Thus, if $b=\delta$, the guard time is reduced to $0.1 \%$ of the burst size. With $10 \mathrm{Gbps}$ ports, the burst size would be 0.5 Mbit (around 150 IP packets as mesured in [23]).

\section{B. Mutual Isolation}

Routes $r$ and $r^{\prime}$ are mutually isolated, denoted by $r \diamond r^{\prime}$, if $r \triangleright r^{\prime}$ and $r \triangleleft r^{\prime}$ (the negation is denoted $r \bowtie r^{\prime}$ ). In addition to disjoint routes, mutual isolation can be achieved between a route that benefits from offset time priority and a route that can reach electrical buffering: Bursts on the former route always "win" the contention whereas the contending ingress bursts are delayed in electrical buffers. It is required that the burst insertion is managed by the ALAP protocol to avoid illegitimate resource access of ingress bursts. Two routes that merge on their first links are also mutually isolated since both can access electrical buffers. Mutual isolation is illustrated on Figure III-B. $C_{1}$ and $C_{2}$ only contend on their first link and are thus isolated. Under the assumption that $\delta \geq b_{c_{3}}, C_{1}$ is isolated from $C_{3}$ if node 1 runs ALAP. On the other side, $C_{3}$ is protected on its first link. On that example, $C_{1}$ is mutually isolated with both $C_{2}$ and $C_{3}$ so that two requests can be served simultaneously (either $C_{1}$ and $C_{3}$ or $C_{1}$ and $C_{2}$ ). Note that if bursts of $C_{2}$ are assigned an extra offset time greater than the size of the bursts of $C_{3}$, then all three connections can be granted with loss less guarantee.

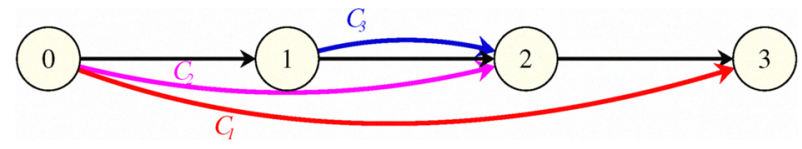

Fig. 6. Mutual Isolation

\section{RWA-OBS FORMULATION}

\section{A. Statement of the Problem}

RWA-OBS is a provisioning problem. For a given traffic matrix, it aims to compute the routing configuration that maximizes either the number of granted requests or the overall amount of traffic granted so that for any two routes $r$ and $r^{\prime}$ that carry flow, $r \diamond r^{\prime}$. The RWA-OBS problem is lowerbounded (throughput wise) by the RWA problem used in WROBS or OCS (see, e.g., [16]) where $r \diamond r^{\prime}$ if and only if $r$ and $r^{\prime}$ are link disjoint. In synchronous networks (SOBS), it can be assumed that $r \diamond r^{\prime}$ for any $r$ and $r^{\prime}$ because the contention avoidance is performed by synchronization. As a result, RWAOBS is upper bounded (throughput wise) by SOBS. Note however that SOBS requires synchronization management that can lead to a resource wastage that is not considered here.

\section{B. Notations}

Denote by $F$ the set of flows, indexed by $f$. For a given $f$, let $o_{f}$ and $d_{f}$ be its origin and destination nodes, $b_{f}$ its bandwidth. $W$ is the set of wavelengths and $\Lambda$ is the set of possible values for the $O T$ extension factor. An OBS route $r$ is associated with a wavelength $\lambda_{r}$ and an $O T$ extension factor $\alpha_{r}$. In this section, $x_{r}$ designates the flow carried by route $r$ and $R_{f, \lambda, \alpha}^{k}$ is a set of $k$ routes from $o_{f}$ to $d_{f}$ on wavelength $\lambda$ with EOT factor $\alpha$. We note $R_{f}^{k}$ the union of all such sets related to connection $f . L$ is the set of links and $\mathrm{TC}_{\ell}$ is the transport capacity of wavelength $w$ on $\ell$. In opposition to the formulation proposed in [13], the $O T$ extension factor is no more global, but associated with a route. As a result, two routes may have different extension factor. The number of OBS routes considered is thus $|W| \times|\Lambda| \times|F| \times k$.

\section{ILP Model}

$$
\begin{aligned}
& z^{\mathrm{OBJ}}=\max \quad \sum_{f \in F} s_{f} b_{f} \quad \text { subject to: } \\
& U_{\ell, w}=\sum_{r \in R_{w}, p_{r} \ni \ell} b_{f(r)} x_{r} \leq \mathrm{TC}_{\ell} \quad \ell \in L, w \in W \\
& x_{r} \leq y_{r} \\
& r \in R \\
& y_{r}+y_{r^{\prime}} \leq 1 \\
& \sum_{r \in c \ell} y_{r} \leq 1 \\
& r, r^{\prime} \in R, r \bowtie r^{\prime} \\
& c \ell \in C \ell \\
& \sum_{r \in R_{f}} x_{r} \geq s_{f} \\
& x_{r} \in[0,1] \\
& y_{r} \in\{0,1\} \\
& s_{f} \in[0,1]
\end{aligned}
$$

where $x_{r}$ represents the fraction of flow $f(r)$ routed on route $r$. Variable $y_{r}$ is linked to $x_{r}$ by (3) so that $y_{r}$ is equal to one if route $r$ is used, zero otherwise. Constraints (6) set the variables $s_{f}$ to the fraction of flow $f$ served with loss-less guarantee. The capacity constraints (2) prevent the wavelength overload (we assume that all wavelengths of a fiber link have the same transport capacity). Constraints (4) prevent non isolated routes to be used simultaneously. Constraints (5) are added to strengthen the LP relaxation. We consider the socalled route conflict graph where each node is associated with 
a route and two nodes are linked if their associated routes are not isolated. A clique $c l$ is a complete sub-graph (any two nodes of the sub-graph are connected). Let $C \ell$ be the set of all maximum (w.r.t. their number of nodes) cliques. The isolation constraints consists in allowing the use of no more than one route per clique. Figure 7 illustrates the improvement of the LP relaxation earned by the clique constraints (or cuts). Therein, the conflict graph exhibits two cliques. As a sequel, no more than two connections can be served, by using one route of each clique ( 2 or 3 and 4 or 5 ). In that example, route 1 should not be used since it belongs to both cliques. Without (5), the LP relaxation affects 0.5 to each route (Figure $7(a))$ whereas constraints (5) avoid the assignment of flow on route 1 (Figure 7(b)).

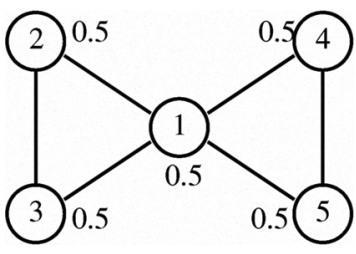

(a) Formulation without (5)

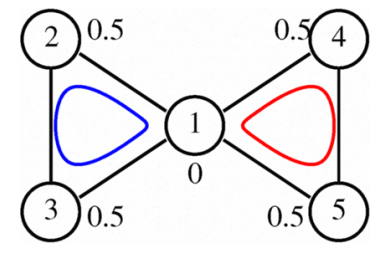

(b) Formulation with (5)
Fig. 7. Isolation Formulation and LP Relaxation

Constraints (10) can be added to take into account the burst insertion delay with the ALAP protocol as discussed in Section II-C: A route can be used if the utilization of its first link is below a ceiling value $\tau \in[0,1]$ (experiments suggest that $\tau=0.6$ is conservative) or if the ingress flow has a major contribution on the load of the link.

$$
y_{r} \leq\left(U_{r[1], \lambda_{r}} \leq 2 b_{f} x_{r}\right)+\left(U_{r[1], \lambda_{r}} \leq \tau \mathrm{TC}_{\ell}\right) \quad r \in R
$$

where $r[1]$ is the first link of route $r$.

\section{Iterative Solution Algorithm}

The number of routes considered directly impact the optimal value of the ILP model: Considering more paths and more EOT values improves the solution, but severely impacts the computing time. To sidestep large computing times, we propose an iterative greedy heuristic (IGH) that consists in iteratively increasing the set of paths (as described by Algorithm 2). At each iteration, one new route per flow is added in the model, the ILP model is solved starting from the solution of the previous iteration and the values of the variables related to the used routes are frozen. Note that the symmetrical nature of the ILP model can be broken by iterating the resolution along the wavelengths as well: Perform the iterative resolution on an increasing number of wavelengths.

After the last iteration, one can either release the values of all the variables and solve the ILP model (which means solve the initial ILP program with a good initial solution) or solve the ILP program only considering the routes that have been used at a given iteration.

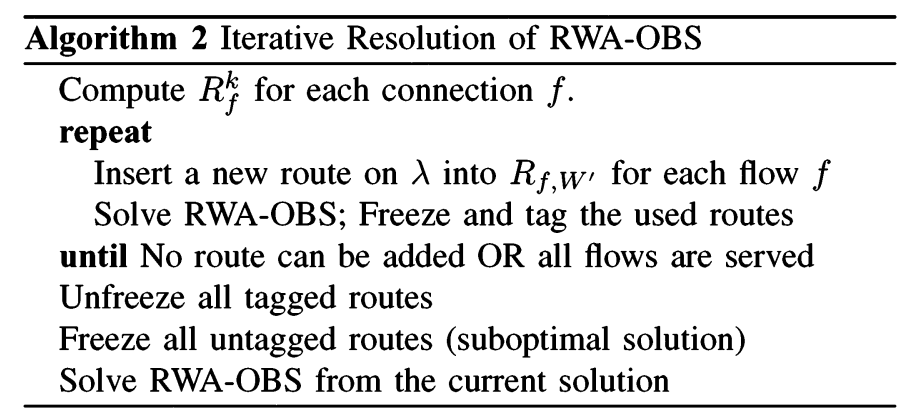

\section{Column Generation Formulation}

Algorithm 2 accommodates the scalability issue due to the number of paths. However, the RWA-OBS problem suffers from its symmetrical structure: There is a huge number of equivalent solutions, all deductible from each other using a wavelength permutation. It is known that integer linear programs (ILPs) with a huge number of equivalent solutions are getting quickly not scalable when, e.g., the number of wavelengths increases in the case of our study. However, turning to a large scale optimization formulation, there is a way to set a model where the number of solutions is greatly reduced, and not equivalent up to a some permutation.

\section{A. Master Problem}

In order to set such a model, let us define a RWA-OBS configuration as a set of routes that can be established on the same wavelength, i.e., that are OBS isolated from each other. Let $\mathcal{C}$ be the set of all possible configurations, indexed by $c \in \mathcal{C}$. Note that, for scalability reasons, the set $R$ of considered routes will be limited to the first $k$ shortest routes, for each pair of source and destination nodes with positive demand.

The model can be written as follows:

$$
\begin{array}{ll}
z^{\text {ов }}=\max _{f \in F} s_{f} b_{f}+\epsilon \sum_{c \in C} \varphi_{c} \quad \text { subject to: } \\
\sum_{c \in \mathcal{C}} z_{c} \leq W & f \in F \\
s_{f}-\sum_{c \in \mathcal{C}} \sum_{r \in R} f_{r}^{c} x_{r}^{c} \leq 0 & c \in C \\
\sum_{r} x_{r}^{c} f_{r}^{c} b_{f}=\varphi_{c} & c \in C, r \in R \\
x_{r}^{c} \leq z_{c} & c \in C \\
z_{c} \in\{0,1\} & f \in F \\
s_{f} \in[0,1] & r \in R \\
x_{r}^{c} \in[0,1] &
\end{array}
$$

where $f_{r}^{c}$ is the maximum fraction of demand $b_{f}$ served by route $r$, on configuration $c$. Denote by $x_{r}^{c}$ the fraction of $f_{r}^{c}$ actually activated and by $s_{f}$ the fraction of the flow $f$ that is accommodated, see constraints (12). Configuration $c$ is activated if $\exists r$ such that $x_{r}^{c} \times f_{r}^{c}>0$, see constraints (14). In that case, $z_{c}=1$. As each configuration is activated on 
different wavelengths, the number of activated configurations must remain lower than $W$, see constraint (11).

\section{B. Pricing Problem}

The number of valid configurations is the number of cliques in the conflict graph. To accommodate this scalability problem, we will solve the LP relaxation of the master problem with a subset of configurations. Two solutions can be envisioned to build the subset: Either we proceed with an off-line enumeration of the configurations, or we generate them on-line. If we go ahead with off-line enumeration, only a subset of configurations can be generated, among the most promising ones, meaning we need a criterion to identify those. The online generation - that is the direction we will move alongrely on the use the column generation techniques to govern an efficient on-line configuration generation. The auxiliary problem (pricing) inserts a new configuration to the incumbent configuration set only if it improves the current value of the objective function, i.e., if it is a so-called augmented configuration. The pricing problem in charge of generating "augmented" configurations aims to maximize the sum of the reduced costs of connections and the objective can be written as follows:

$$
\max \quad-u_{0}+\sum_{f} u_{f} s_{f}+\varepsilon\left(\sum_{f \in F} \sum_{r \in R_{f}} x_{r} b_{f}\right)
$$

where $u_{0}$ and $u_{f}$ are the dual variables respectively associated with constraints (11) and (12). The last term helps to add as much traffic as possible in the best configuration. For example, a route isolated from every route should appear in every configuration whether served or not.

The set of constraints is the same than in the original model described in Section IV, but the set of routes is restricted to a single wavelength (its cardinality thus decreases down to $|F| \times|\Lambda| \times k)$.

$$
\begin{array}{ll}
\sum_{f} \sum_{r \in R_{f}, r \ni \ell} b_{f} x_{r} \geq \mathrm{TC}_{\ell} & r \in R \\
\sum_{r \in c \ell} y_{r} \leq 1 & \ell \in L \\
\sum_{r \in R_{f}} x_{r}=s_{f} & c \ell \in C \ell \\
s_{f} \in[0,1] & f \in F \\
y_{r} \in\{0,1\}, x_{r} \in[0,1] & f \in F
\end{array}
$$

\section{ILP Solution}

The column generation stops when the pricing problem cannot generate a column that improves the LP relaxation of the master problem. We next solve the ILP master, i.e., with $z_{c}$ being 0-1 variables, with the current subset of configurations. Nevertheless, reaching the optimality of the LP relaxation does not ensure that the subset of configurations leads to the optimal solution of the MIP version of the problem. This phenomenon

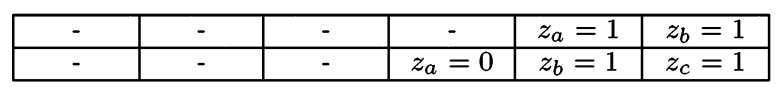

\begin{tabular}{|c|c|c|c|c|c|}
\hline$z_{a}=0$ & $z_{b}=0$ & $z_{c}=0$ & $z_{d}=0$ & $z_{e}=1$ & $z_{f}=1$ \\
\hline$z_{b}=0$ & $z_{c}=0$ & $z_{d}=0$ & $z_{e}=0$ & $z_{f}=1$ & $z_{g}=1$ \\
\hline
\end{tabular}

TABLE I

TABU ILLUSTRATION WITH 3 WAVELENGTHS

can be illustrated by Figure 7. Let us assume that routes 2 and 4 serve the flow $f$ and routes 3 and 5 serve the flow $f^{\prime}$. Only one wavelength is available. The optimal value is 2 for both the LP and ILP programs. An optimal LP solution is with configurations $c=[0,1,0,1,0]$ and $c^{\prime}=[0,0,1,0,1]$ if $z_{c}=z_{c^{\prime}}=0.5$, whereas the ILP optimal value with this set of configurations is 1 .

To force the LP master problem to stay with feasible integer solutions, we propose to freeze some configurations in the master problem to integer value. The management of the frozen configuration is done via a Tabu list. The size of the Tabu list is set to $2 \times|W|$. If the LP relaxation solution involves more than $|W|$ wavelengths, then the configuration $c$ that handles the highest amount of traffic is added to the Tabu list and $z_{c}$ is set to 1 . The content of the Tabu list is swapped. The $W-1$ earliest inserted configurations are frozen as used whereas the $W+1$ last inserted ones are frozen as unused until they are discarded from the Tabu list.

Table I illustrates the Tabu list management with $|W|=$ 3. $z_{a}$ and $z_{b}$ are frozen as used configurations. When $z_{c}$ is inserted, the Tabu list content is swapped and $z_{a}$ is frozen as unused whereas $z_{c}$ is frozen as used. $z_{a}$ cannot be used until it is discarded from the Tabu list (at the insertion of $z_{g}$ ).

\begin{tabular}{l}
\hline Algorithm 3 Column Generation Tuning \\
\hline repeat \\
Solve the master LP relaxation \\
if number of used configurations $>W$ then \\
add the most useful configuration in the Tabu list \\
Solve the master LP relaxation \\
end if \\
Solve the pricing problem \\
Add the new configuration in the master problem \\
until Time limit exceeds \\
Release all configurations; Solve the master ILP problem \\
\hline
\end{tabular}

\section{EXPERIMENTAL RESULTS}

In this section, we report numerical results obtained on the NSF network (14 nodes and 23 links) and the New Jersey LATA network (11 nodes 23 links) available on [24]. We assume $b<\delta$ as discussed in Section III. The load is equal between each pair of nodes and expressed as a ratio of the wavelength transport capacity (10 Gbps).

\section{A. RWA-OBS vs. WR-OBS and SOBS}

We first compare the normalized throughput of loss-less guaranteed traffic handled with RWA-OBS, WR-OBS and 
SOBS. In our experiments, we relaxed the synchronization constraints that can lead to resource wastage. The results thus reflect the best possible performance of SOBS.

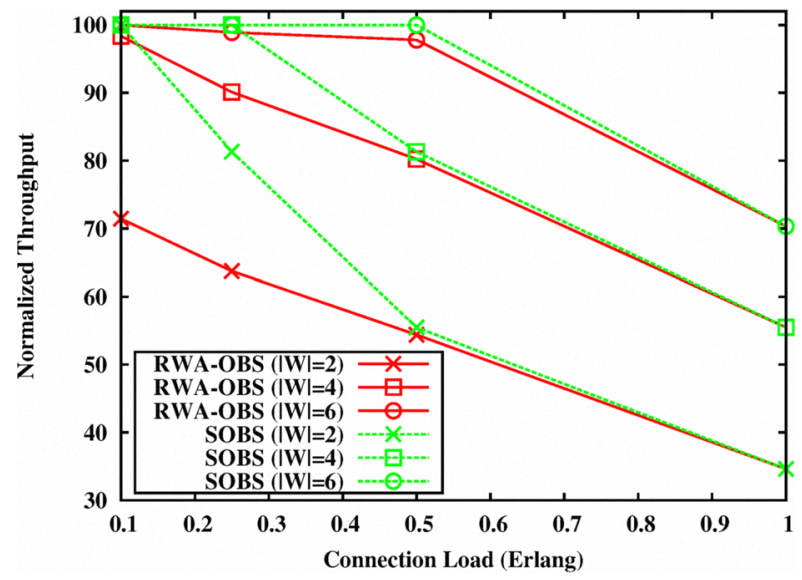

(a) NSF Network

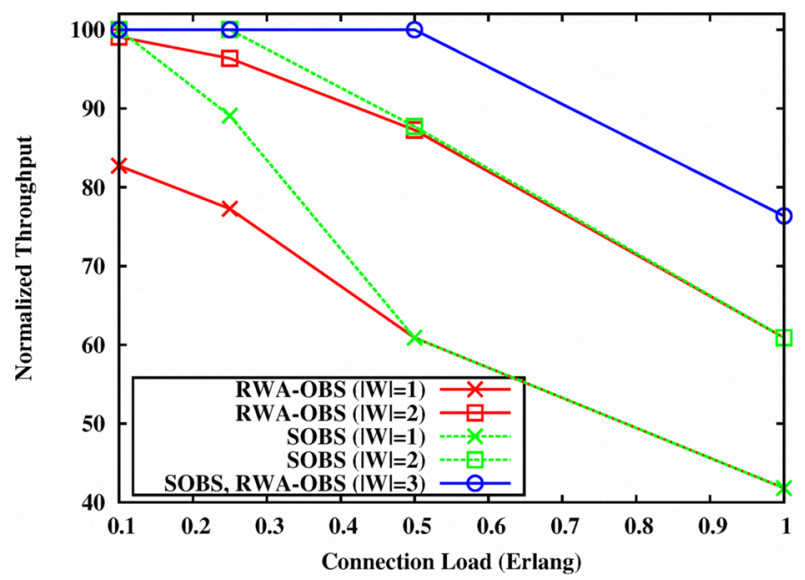

(b) New Jersey LATA Network

Fig. 8. Normalized Throughput

With WR-OBS, multiplexing is prohibited so that the throughput is equal to the throughput obtained with SOBS and RWA-OBS in the case where the load of each flow matches the wavelength transport capacity. With softer flows, multiplexing is possible with RWA-OBS and SOBS, so the normalized throughput increases and therefore, WR-OBS is outperformed. With SOBS, contention is avoided by external synchronization mechanisms so that any two flows can be multiplexed under the capacity constraint. The multiplexing potential of SOBS is thus maximum and the performances of RWA-OBS are in between those of WR-OBS and SOBS because the set of configurations that guarantee flow isolation is restricted to those identified in Section III. On the ring topology, the use of the $O T$ extension factor elevates the throughput at the level of the one of SOBS. Figure 8(a) reports the results on the NSF network. SOBS outperforms RWA-OBS with soft connection load ( $10 \%$ of the wavelength granularity) and less than 4 wavelengths. However, if the load of the requests increases, the multiplexing potential of SOBS is reduced and the gap with RWA-OBS decreases: With request granularity of half the connection granularity, no more than $3 \%$ of the throughput is lost with RWA-OBS. The same observation can be done on the NJ-LATA network (Figure 8(b)): The gap between SOBS and RWA-OBS is negligible if connections request more than half the wavelength capacity, but increases when the connection load decreases.

\section{B. Efficient Solution}

The exact model presented in Section VI-A is heavy and requires large computation time. In this section, we compare the computation time and the quality of the solutions obtained with the iterative greedy heuristic (IGH) and the column generation approach (CG-RWA-OBS). Table II reports the computing times and the gaps between the heuristic values and the optimal values obtained with the ILP formulation. From the results, we can identify "easy instances" and "hard instances". Instances with a connection load of 0.5 Erlang are more difficult to solve because the symmetry of the problem is increased. If the load is equal to the wavelength transport capacity, then the wavelength capacity constraints prevent multiplexing and constraints (4) and (5) can be relaxed. On the opposite, the capacity constraints are useless if the load is equal to 0.1 Erlang. The instances with $W=4$ are also difficult to solve because they are the largest instances where the full provisioning is impossible. The exact solution of the ILP formulation is highly dependent on the number of wavelengths. Indeed, the optimal solutions of the difficult instances could not be obtained in reasonable time. IGH clearly outperforms the ILP formulation in terms of computing times but the quality of the IGH solutions may not be satisfactory, especially for "hard instances" (with a 5\% to $7.5 \%$ gap). CGRWA-OBS improves the quality of the solutions, often up to the optimal solution. Computing times are comparable for easy instances and although computing times are longer for "hard instances", they remain reasonnable, especially when compared with those of the exact ILP model.

\section{ALAP Delay Reduction}

The addition of constraints (10) offers a control on the compromise between delay and throughput. As the model is significantly enlarged, we used CG-RWA-OBS to compute the routing tables on an instance with $|W|=4$ and a request load of 2 Gbps. Table III reports simulation measurements of the throughput and the insertion delay for various values of the $\tau$ threshold parameter. As the threshold reduces the bandwidth availability, the grade of service of the RWA-OBS model is reduced for smaller values of $\tau$. Consequently, the throughput as evaluated by simulation decreases, but the insertion delay is drastically reduced. 


\begin{tabular}{|c|c|c|c|c|}
\hline & $|W|$ & \multicolumn{3}{|c|}{ Load (Erlang) } \\
\hline & & 0.1 & 0.5 & 1 \\
\hline ILP & $\begin{array}{r}2 \\
4 \\
6 \\
8 \\
10\end{array}$ & $\begin{array}{r}36 \\
89,161 \\
5,274 \\
9,094 \\
20,133\end{array}$ & $\begin{array}{r}>2 \text { weeks } \\
>2 \text { weeks } \\
>2 \text { weeks } \\
123,300 \\
106,531\end{array}$ & $\begin{array}{r}1 \\
174 \\
6,820 \\
5,703 \\
8,236 \\
\end{array}$ \\
\hline IGH & $\begin{array}{r}2 \\
4 \\
6 \\
8 \\
10\end{array}$ & $\begin{array}{r}3 \\
7 \\
22 \\
9 \\
12\end{array}$ & $\begin{array}{r}458 \\
683 \\
6,628 \\
2,200 \\
752\end{array}$ & $\begin{array}{r}1 \\
3 \\
5 \\
7 \\
19\end{array}$ \\
\hline Gap $=\frac{z^{\mathrm{ILP}}-z^{\mathrm{H}}}{z^{\mathrm{ILP}}}$ & $\begin{array}{r}2 \\
4 \\
6 \\
8 \\
10 \\
\end{array}$ & $\begin{array}{r}2.4 \% \\
7.5 \% \\
0.6 \% \\
0 \\
0 \\
\end{array}$ & $\begin{array}{c}5.0 \% \\
6.1 \% \\
5.1 \% \\
1.1 \\
0\end{array}$ & $\begin{array}{rr} & 0 \\
1.0 \% \\
0.8 \% \\
3.4 \% \\
3.1 \% \\
\end{array}$ \\
\hline CG - RWA OBS & $\begin{array}{r}2 \\
4 \\
6 \\
8 \\
10\end{array}$ & $\begin{array}{r}6 \\
5,349 \\
319 \\
6 \\
6\end{array}$ & $\begin{array}{r}24,290 \\
67,878 \\
76,822 \\
447 \\
56\end{array}$ & $\begin{array}{r}1 \\
2 \\
2 \\
3 \\
3,223 \\
\end{array}$ \\
\hline $\mathrm{Gap}=\frac{z^{\mathrm{ILP}}-z^{\mathrm{CG}}}{z^{\mathrm{ILP}}}$ & $\begin{array}{r}2 \\
4 \\
6 \\
8 \\
10\end{array}$ & $\begin{array}{r}0 \\
1.2 \% \\
0.5 \% \\
0 \\
0\end{array}$ & $\begin{array}{r}1.2 \% \\
0 \\
0 \\
1.1 \% \\
0\end{array}$ & $\begin{array}{r}0 \\
0 \\
0 \\
0 \\
0.75 \% \\
0.70 \%, 0\end{array}$ \\
\hline
\end{tabular}

TABLE II

SOLUTION SCHEME COMPARISON

\begin{tabular}{|l|c|c|c|}
\hline Threshold $(\tau)$ & 0.2 & 0.6 & 1 \\
\hline Throughput $(\mathrm{Gbps})$ & 226 & 256 & 286 \\
\hline Delay $(\mathrm{ms})$ & 0.15 & 1.0 & 1.7 \\
\hline
\end{tabular}

TABLE III

BURST INSERTION DELAy REDUCTION

\section{CONCLUSION}

In this paper, we have recalled the possibility of asynchronous loss less transfer with statistical multiplexing. The new RWA-OBS formulation improves on the first RWAOBS formulation described in [15]: (i) The LP relaxation is improved by the clique constraints ; (ii) the $O T$ extension factor can be tuned for each route so that the flexibility and consequently, the throughput is increased and (iii) an additional constraint is included to control the compromise between the grade of service and the burst insertion delay.

RWA-OBS offers an intermediate multiplexing potential between WR-OBS and SOBS but can achieve similar performances as SOBS if the request granularity is above half the wavelength transport capacity.

We proposed an iterative heuristic that can quickly provides acceptable solutions. The column generation approach, however, improves the quality of the solutions for a reasonable increase of the computing times. The flexibility of the column generation model allows for an easy addition of a constraint that effectively compromise between the burst insertion delay (increased by the ALAP burst insertion protocol) and the throughput.

\section{REFERENCES}

[1] M. Yoo, M. Jeong, and C. Qiao, "A new optical burst switching (OBS) protocol for supporting quality of service," in SPIE, vol. 3531, November 1998, pp. 396-405.

[2] A. Zalesky, H. Vu, Z. Rosberg, E. Wong, and M. Zukerman, "OBS contention resolution performance," Performance Evaluation, vol. 64, no. 4, pp. 357-373, 2007.

[3] M. Phùng, K. Chua, G. Mohan, M. Motani, and T. Wong, "The streamline effect in OBS networks and its application in load balancing," in 2nd International Conference Broadband Networks, October 2005, pp. $304-311$.

[4] L. Yang and G. Rouskas, "Adaptive path selection in optical burst switched networks," Journal of Lightwave Technology, vol. 24, pp. 3002-3011, 2006.

[5] C. Qiao, W. Wei, and X. Liu, "Extending generalized multiprotocol label switching (GMPLS) for polymorphous, agile, and transparent optical networks (PATON)," IEEE Communications Letters, vol. 44, pp. 104114, December 2006.

[6] T. Coutelen, H. Elbiaze, and B.Jaumard, "Performance comparison of OCS and OBS switching paradigms," in 7th International Conference on Transparent Optical Networks (ICTON), July 2005, pp. 212-215.

[7] Y. Fan and B. Wang, "Exploring node light-splitting capability for burst grooming in optical burst switched networks," Photonic Network Communications, vol. 14, no. 2, pp. 209-222, 2007.

[8] V. Lopez, J. Garcia-Dorado, J. Hernandez, and J. Aracil, "Performance comparison of scheduling algorithms for IPTV traffic over polymorphous OBS routers," 2007, pp. 1-6.

[9] Synchronous Optical Burst Switching, 2004.

[10] O. Yu, M. Liao, and Y. Cao, "Multi-granular stream optical burst switching," in BROADNETS, 2006, pp. 1-10.

[11] J. Angelopoulos, K. Kanonakis, G. Koukouvakis, H. Leligou, C. Matrakidis, T. Orphanoudakis, and A. Stavdas, "An optical network architecture with distributed switching inside node clusters features improved loss, efficiency, and cost," Journal of Lightwave Technology, vol. 25, pp. 1138 - 1146, May 2007.

[12] M. Yoo and C. Qiao, "Supporting multiple classes of services in IP over WDM networks," in IEEE Global Telecommunications Conference - GLOBECOM, 1999, pp. 1023-1027.

[13] T. Coutelen, G. Hébuterne, and B. Jaumard, "Core OBS traffic properties and behavior," GERAD, Les Cahiers du GERAD G-09-43, May 2009.

[14] M. Yoo, C. Qiao, and S. Dixit, "A comparative study of contention resolution policies in optical burst switched WDM networks," in Conference on Terabit Optical Networking, vol. 4213, 2000, pp. 124-135.

[15] T. Coutelen, G. Hébuterne, and B. Jaumard, "An OBS RWA formulation for asynchronous loss-less transfer in OBS networks," in IEEE Workshop on High Performance Switching and Routing (HPSR), 2009.

[16] B. Jaumard, C. Meyer, and B. Thiongane, "ILP formulations for the RWA problem for symmetrical systems," in Handbook for Optimization in Telecommunications, P. Pardalos and M. Resende, Eds. Kluwer, 2006, ch. 23, pp. 637-678.

[17] G. Fiche and G. Hébuterne, Communicating Systems \& Networks: Traffic \& Performance. Kogan Page Science, 2004.

[18] C. Gauger, "Dimensioning of FDL buffers fr optical burst switching nodes," in Conference on Next Generation Optical Network Design and Modelling - ONDM, 2003, pp. 117-132.

[19] G. Gurel and E. Karasan, "Using multiple per egress burstifiers for enhanced TCP performance in OBS networks," Photonic Network Communications, vol. 17, pp. 105-117, April 2009.

[20] T. Coutelen, G. Hébuterne, and B. Jaumard, "Is it worth to keep an all optical OBS data plane?" in Canadian Conference on Electrical and Computer Engineering (CCECE), 2009.

[21] Y. Sun, T. Hashiguchi, V. Q. Minh, X. Wang, H. Morikawa, and T. Aoyama, "Design and implementation of an optical burst-switched network testbed," IEEE Communications Magazine, vol. 43, pp. S48S55, November 2005.

[22] H. Guo, Z. Lan, J.Wu, Z. Gao, X. Li, J. Lin, and Y. Ji, "A testbed for optical burst switching network," in Optical Fiber Communication Conference, vol. 5, 2005

[23] S. McCreary and K. Claffy, "Trends in wide area IP traffic patterns," ITC Specialist Seminar, 2000.

[24] www.optical-network.com/topology.php. 